\title{
Intususcepción como complicación de bypass en Y de Roux en pacientes embarazadas, una complicación infrecuente. Reporte de caso y revisión de la literatura
}

\author{
Intussusception as a complication of Roux-en-Y-gastric bypass in pregnant patients, an \\ uncommon complication. Case report and literature review
}

\section{Ricardo J. Salinas-Garza1 , Alan A. Garza-Cantú2, José E. Martínez-Escalante ${ }^{1 *}$, Meri Yeghiazaryan ${ }^{1}$ y Tania M. Jiménez-Molina ${ }^{3}$}

${ }^{1}$ Servicio de Cirugía General, Christus Muguerza, Hospital Alta Especialidad, Monterrey, Nuevo León;²Servicio de Coloproctología, Hospital General de México, Ciudad de México; ${ }^{3}$ Servicio de Cirugía, Hospital Ángeles Mocel, Ciudad de México. México

\section{Resumen}

La obesidad es actualmente un problema de salud pública de ámbito internacional. Es considerada como la segunda causa de muerte prevenible en México, después del tabaquismo, según la Organización Mundial de la Salud. Actualmente, las opciones quirúrgicas son la gastrectomía en manga, el bypass gástrico o derivación gástrica, la banda gástrica y el bypass gástrico en omega, cada una con sus indicaciones. Se presenta el caso clínico de una paciente con intususcepción yeyuno-yeyunal secundaria a bypass gástrico en el embarazo.

Palabras clave: Bariatría. Bypass gástrico. Embarazo. Intususcepción. Obesidad mórbida.

\begin{abstract}
Obesity is currently an international public health problem. It is considered the second preventable cause of death in Mexico, after smoking, according to the World Health Organization. Currently, surgical options are sleeve gastrectomy, gastric bypass or gastric derivation, gastric banding, and omega gastric bypass, each with specific indications. The objective of the article is to present the clinical case of a patient with jejuno-jejunal intussusception secondary to gastric bypass in pregnancy.
\end{abstract}

Key words: Bariatrics. Gastric bypass. Pregnancy. Intussusception. Morbid obesity.

\section{Antecedentes}

La obesidad es uno de los problemas de salud más importantes del mundo moderno. La Organización
Mundial de la Salud la considera como una epidemia global, siendo la segunda causa de muerte prevenible después del tabaquismo, y además reconoce los costos elevados de su atención, que requiere el $2-7 \%$ de los presupuestos nacionales para la salud ${ }^{1}$.

\footnotetext{
Correspondencia:

*José E. Martínez-Escalante

Sacramento, 205a

Col. Mitras Centro

Fecha de recepción: 04-02-2020

C.P. 64460 , Monterrey, N.L., México

E-mail: emmanuelmtz@ @otmail.es

Cir Cir. 2020;88(S2):9-12

Contents available at PubMed www.cirugiaycirujanos.com 0009-7411/@ 2020 Academia Mexicana de Cirugía. Publicado por Permanyer. Este es un artículo open access bajo la licencia CC BY-NC-ND (http://creativecommons.org/licenses/by-nc-nd/4.0/).
} 
La primera operación para tratar la obesidad fue realizada por el Dr. Henrikson en 1950, quien realizó una resección extensa de intestino delgado con el objetivo de disminuir la superficie de absorción intestinal, motivando a otros cirujanos a enfocar sus intereses en otros tipos de bypass. El bypass yeyuno-cólico fue el primero en realizarse, pero provocó una importante pérdida de líquidos y electrolitos, así como falla hepática grave, por lo que tuvo que revertirse.

En 1954, el Dr. Arnold Kremer, de la Universidad de Minneapolis, junto con el Dr. John H. Linner y el Dr. Charles Nelson, publicaron los resultados de la cirugía de bypass yeyuno-ileal para el tratamiento de la obesidad mórbida, sentando las bases para los procedimientos antes mencionados. Se obtuvieron resultados desalentadores, ya que dicha cirugía se asociaba a grandes complicaciones, como el síndrome de asa ciega debido a estasis y sobrecrecimiento bacteriano y hemorragia del intestino excluido, continuando así la búsqueda del procedimiento ideal.

Fue en 1966 cuando el Dr. Edward E. Manson realizó la primera operación de derivación gástrica, naciendo así las operaciones bariátricas malabsortivas actualmente utilizadas ${ }^{2-5}$.

Presentamos un caso clínico de complicación tardía de bypass gástrico en $Y$ de Roux (BGYR) en una paciente embarazada.

\section{Caso clínico}

Mujer de 27 años que cursaba embarazo de 32.6 semanas con antecedente de BGYR por laparoscopía realizado un año y medio antes del cuadro, la cual se presentó al departamento de urgencias de otra institución por una historia de dolor abdominal de 24 horas de evolución, de inicio insidioso, tipo cólico, de moderada intensidad, localizado en el mesogastrio, agravado con la ingestión de alimentos, atenuado parcialmente con analgésicos, acompañado de hiporexia y náuseas, sin irradiaciones. Se dio de alta con manejo analgésico y dieta astringente, con datos de alarma. Progreso 12 horas posterior a la revisión inicial con aumento en la intensidad del dolor, volviéndose de intensidad grave y localizado en los cuatro cuadrantes, sin atenuación con los analgésicos, agregándose hematemesis en dos ocasiones, motivo por el cual acudió nuevamente al departamento de urgencias, donde se encontró en la exploración física abdomen distendido con peristalsis disminuida y datos de irritación peritoneal. Se decidió realizar resonancia magnética abdominal que evidenció una imagen

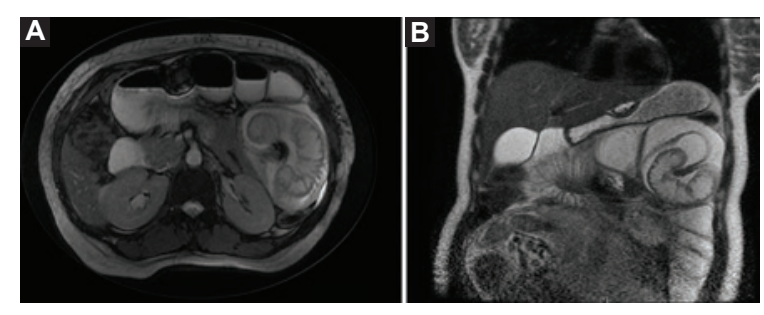

Figura 1. Resonancia magnética de abdomen que identifica la intususcepción de un asa de intestino delgado. A: corte transversal. B: corte coronal.

sugestiva de hernia interna o intususcepción intestinal (Fig. 1). Se trasladó a nuestro hospital, donde se programó para laparotomía urgente. Previo a la cirugía se realizó interconsulta con el servicio de ginecología y obstetricia, quienes realizaron un registro cardiotocográfico sin evidenciar sufrimiento fetal. Se decidió interrumpir el embarazo por medio de cesárea y posteriormente, en el mismo tiempo quirúrgico, se realizó abordaje y exploración abdominal por parte de cirugía general. El producto fue categorizado con Apgar 6-8.

Se llevó a cabo una ampliación de la incisión de laparotomía media realizada por el servicio de ginecología y obstetricia, observando asas de intestino delgado dilatadas con área de transición localizada a $110 \mathrm{~cm}$ del ligamento de Treitz a nivel de la yeyuno-yeyuno anastomosis, encontrando intususcepción de asa común (Fig. 2 A). Se trató de reducir, pero se fracasó, por lo que se realizó enterotomía y, al observar necrosis intestinal transmural en el intestino con intususcepción (Fig. 2 B), se decidió realizar una resección de intestino delgado de aproximadamente $60 \mathrm{~cm}$, con posterior entero-entero anastomosis con grapadora lineal, cartucho azul de $60 \mathrm{~mm}$, a $20 \mathrm{~cm}$ de la yeyuno-yeyuno anastomosis (Fig. $3 \mathrm{C}$ ), previa descompresión del intestino delgado. Se realizó el cierre por planos y la paciente pasó a recuperación sin eventualidades.

Evolucionó satisfactoriamente, canalizando gases a las 48 horas y con tolerancia a la dieta a las 72 horas posoperatorias, sin datos de respuesta inflamatoria sistémica. Se egresó al sexto día de posoperatorio. El producto se da de alta conjunta sin complicaciones.

\section{Discusión}

Entre los puntos más importantes para destacar de este caso clínico se encuentra la necesidad de un alto índice de sospecha ante la presentación de un paciente con antecedente de cirugía bariátrica con dolor abdominal a urgencias, así como la importancia del 

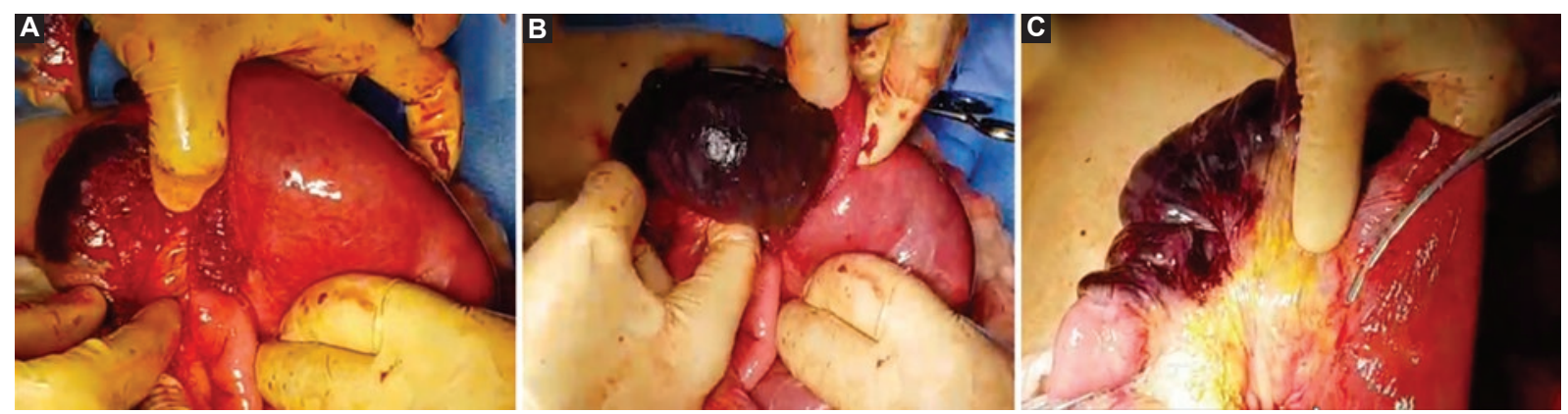

Figura 2. Asa de intestino con intususcepción yeyuno-yeyunal demostrando isquemia transmural. A: identificación del asa. B: reducción del asa. C: resección del asa.

manejo multidisciplinario para optimizar resultados. En nuestro caso, al decidir la realización de estudios de imagen durante el embarazo puede hacerse con radiografías simples o incluso recurrir a una tomografía computarizada de abdomen sin mayor riesgo de daño al producto en su tercer trimestre de gestación. Otro aspecto es la necesidad de interrupción del embarazo, que se ha descrito en diferentes series; en ausencia de sufrimiento fetal o inestabilidad de la madre o predicción de complicaciones a corto plazo, se debe optar por no interrumpir el embarazo si no se encuentra a término.

Este caso reporta la presencia de dolor abdominal inespecífico en una paciente embarazada con antecedente de BGYR, siendo la intususcepción intestinal uno de los diagnósticos diferenciales a descartar. A pesar de su rara frecuencia, debe ser parte de la sospecha diagnóstica ante dicha presentación, pues si se retrasan su diagnóstico y tratamiento la morbimortalidad maternofetal aumenta exponencialmente ${ }^{6}$.

Entre las complicaciones tardías más frecuentes de la cirugía bariátrica se encuentran la estenosis, la obstrucción por adherencias, las úlceras marginales, las hernias internas y la intususcepción?.

La obstrucción intestinal ocurre en un $9.7 \%$ de los pacientes y es más frecuente después de un procedimiento laparoscópico (1.6-9.7\%) que de uno abierto (0.3-1\%). La colocación del asa en $Y$ de Roux retrocólica se asocia con una mayor probabilidad (4.5$7.0 \%$ ) de obstrucción de intestino delgado que la técnica antecólica $(0.42-2 \%)^{2,8}$. La intususcepción después de un procedimiento bariátrico tiene un rango de presentación del $0.1-0.3 \%$ después de un $\mathrm{BGYR}^{1,6,9}$, y puede llegar a asociarse a un alta mortalidad materna y perinatal, de un $6 \%$ y un $26 \%$, respectivamente ${ }^{6}$.
La posible etiología de la intususcepción descrita por Hocking en 1991 teoriza acerca del origen afirmando que la sutura mecánica actúa como un «punto principal» para la invaginación, aunado a que la actividad peristáltica desordenada en la extremidad de Roux podría crear áreas adyacentes de alta y baja presión, predisponiendo a la intususcepción, causando la obstrucción intestinal y disminuyendo el flujo sanguíneo al mesenterio ${ }^{1,5,6}$. También se menciona en otro artículo que la etiología puede ser multifactorial y ocurre por la combinación de la disrupción de los marcapasos naturales y la disminución del grosor del mesenterio, creando una zona de inestabilidad alrededor del asa de Roux y la yeyuno-yeyunostomía, y con la combinación de estos factores se puede predisponer a la intususcepción ${ }^{5,7,9}$.

El tiempo entre el BGYR y la aparición de los síntomas en la población no embarazada es variable, generalmente entre 1 y 3 años después de la cirugía. El tiempo medio de manifestación posterior al BGYR fue de 4 años y la edad gestacional media fue de 27.42 semanas. Los síntomas que se pueden presentar, según describen otros reportes de casos en siete pacientes, son la presentación con dolor abdominal intermitente con presentación aguda o de varios días, acompañándose de náuseas ${ }^{1,7}$. Para el diagnóstico, realizar una anamnesis detallada es clave, destacando los antecedentes quirúrgicos, con una exploración física dirigida, y ante la duda o sospecha de algún cuadro de obstrucción intestinal secundario a una intususcepción hay que solicitar estudios de imagen para auxiliar en el diagnóstico oportuno para su manejo. La tomografía computarizada contrastada confirma el diagnóstico en un $80 \%$ de los casos, considerando el riesgo-beneficio de la exposición de radiación al feto y la madre ${ }^{1-6}$. 
Dentro de las opciones de tratamiento se puede optar por una laparoscopia diagnóstica, en caso de ser tolerado el neumoperitoneo, o una cirugía abierta, siempre vigilando la condición fetal. El tratamiento deberá ser individualizado dependiendo de la edad de la paciente, la edad gestacional y la vitalidad del intestino afectado, dentro de los cuales se puede optar por resección y anastomosis ${ }^{6}$. El trabajo de Stephenson, et al. ${ }^{10}$ incluyó 3022 pacientes que se sometieron a BGYR, de los cuales 12 (0.4\%) presentaron intususcepción posterior. Entre las opciones quirúrgicas que utilizaron en dicha serie se encuentran la reducción y la revisión de la yeyuno-yeyuno anastomosis, la imbricación/plicatura de la yeyuno-yeyuno anastomosis, disminuyendo su área de superficie, y la resección con anastomosis ${ }^{4,10,11}$. En nuestro caso se optó por la resección del intestino afectado debido a la necrosis intestinal transmural en el intestino con intususcepción, con una anastomosis mecánica, cursando un posoperatorio sin complicaciones, así como también con una buena evolución hacia la mejoría.

El retraso en la intervención quirúrgica en presencia de obstrucción de intestino delgado puede aumentar la morbimortalidad materna y fetal. El tratamiento quirúrgico dentro de las 48 horas del cuadro clínico tiene un $10 \%$ de mortalidad, mientras que después de 48 horas del diagnóstico aumenta al $50 \%$. Solo se ha informado de una muerte neonatal por enterocolitis necrotizante asociada a neumonía materna ${ }^{1,12,13}$.

\section{Conclusión}

El conocimiento clínico de las complicaciones bariátricas, así como el alto índice de sospecha, deben tomarse en cuenta siempre que se presente algún paciente con este antecedente y dolor abdominal agudo, especialmente si es sugestivo de oclusión intestinal. En caso de que el diagnóstico sea franco, los estudios de imagen se pueden obviar, ya que en ocasiones esto retrasaría el manejo quirúrgico definitivo con el consecuente aumento en la morbimortalidad. En caso de tratarse de pacientes embarazadas, deberá consultarse a un médico especialista en ginecología y obstetricia para un manejo conjunto, teniendo en cuenta que ante la presentación clínica de dolor abdominal agudo con antecedente de BGYR se trata de una oclusión intestinal hasta demostrar lo contrario, y por último que, de ser necesario someter a la paciente a una cesárea, se debe revisar la totalidad del intestino delgado para descartar su involucro.

\section{Conflicto de intereses}

Los autores declaran no tener conflicto de intereses.

\section{Agradecimientos}

Al Hospital Christus Muguerza Alta Especialidad por permitirnos la utilización de los recursos para la elaboración de este manuscrito con fines educativos.

\section{Responsabilidades éticas}

Protección de personas y animales. Los autores declaran que para esta investigación no se han realizado experimentos en seres humanos ni en animales.

Confidencialidad de los datos. Los autores declaran que han seguido los protocolos de su centro de trabajo sobre la publicación de datos de pacientes.

Derecho a la privacidad y consentimiento informado. Los autores han obtenido el consentimiento informado de los pacientes y/o sujetos referidos en el artículo. Este documento obra en poder del autor de correspondencia.

\section{Bibliografía}

1. González JR, Márquez JG, Limón Aguilar JL, Márquez Aldama G. Bypass gástrico. Tratamiento quirúrgico de la obesidad mórbida. Rev Hosp Jua Mex. 2005;72:153-60. Disponible en: http://www.medigraphic. com.

2. Henrikson V. Can small bowel resection be defended for therapy for obesity? Obes Surg. 1994;4:54-5.

3. Angrisani L, Procedures R, De Luca M, Formisano G, Santonicola A, editores. Bariatric and metabolic surgery. Italia: Springer-Verlag; 2017.

4. Kremen AJ. An experimental evaluation of the nutritional importance of proximal and distal small intestine. Ann Surg. 1954;140:439-44.

5. Masson EE. Gastric bypass in obesity. Surg Clin North Am. 1967;47:1345-51.

6. Boccalatte LA, Achaval Rodríguez J, Beskow A, Cavadas D, Fernando W. Intussusception as a complication of bariatric surgery in pregnant patients: report of one case and revision of the literature. J Surg Case Reports. 2017;2017(10):rjx189.

7. Herron DM. Bariatric Surgery Complications and Emergencies. Springer. 1a. Edición. New York, EUA, 2016.

8. Still C, Sarwer DB. The ASMBS Textbook of Bariatric Surgery. Springer. 1a. Edición. New York, EUA, 2014.

9. Blackstone RP. Bariatric Surgery Complications. Springer. 1a. Edición. New York, EUA, 2017.

10. Stephenson D, Moon RC, Teixeira AF, Jawad MA. Intussusception after Roux-en-Y gastric bypass. Surg Obes Relat Dis. 2014;10:666-70.

11. Wax JR, Pinette MG, Cartin A. Roux-en-Y gastric bypass-associated bowel obstruction complicating pregnancy - an obstetrician's map to the clinical minefield. Am J Obstet Gynecol. 2013;208:265-71.

12. Singla S, Guenthart BA, May L, Gaughan J, Meilahn JE. Intussusception after laparoscopic gastric bypass surgery: an underrecognized complication. Minim Invasive Surg. 2012;2012:464853.

13. Bokslag A, Jebbink J, De Wit L, Oudijk M, Ribbert L, Tahri S, et al. Intussusception during pregnancy after laparoscopic Roux-en-Y gastric bypass. BMJ Case Rep. 2014;2014:bcr2014205357. 